\title{
COORDINATING OPENNESS TO DIVERSE AND CONTESTING CONTRIBUTIONS IN ONLINE COMMUNITIES
}

\author{
ALJONA ZORINA \\ Leeds University Business School, UK \\ Leeds, LS2 9JT UK \\ Email: a.zorina@leeds.ac.uk \\ JOANA PEREIRA \\ Leeds University Business School, UK

\section{INTRODUCTION}

Online communities (OCs) have been discussed as one of the most prominent and successful innovation actors in the $21^{\text {st }}$ century (Baldwin and Von Hippel, 2011; Benner and Tushman, 2015; O'Mahony and Lakhani, 2011). The example of Apache, Linux, and Wikipedia, amongst others, show that collectives that communicate and engage in repeated interactions can efficiently coordinate to create socio-economic value (Benkler, 2002). OCs are "virtual places" where individuals with similar needs, problems, or interests can interact, exchange and build knowledge and ultimately generate innovation (Dahlander and Frederiksen, 2012; Franke and Shah, 2003; Jeppesen and Frederiksen, 2006; Lüthje, 2004; Preece, 2000; Tardini and Cantoni, 2005). While OCs are sites of innovation and creativity in contemporary organizing (e.g. Chesbrough, 2006; von Hippel and von Krogh, 2006), they also face the challenge of coordinating inputs from heterogeneous actors (e.g. individuals and organizations) (Benkler, 2002; Barret et al., 2013; Faraj et al., 2016; Faraj et al., 2011; von Krogh et al., 2012). Therefore, the dilemma of openness to members and their contributions versus a need for coordination is particularly relevant in open OCs.

Differently from traditional bureaucratic organizations, where reliance on a common goal is fundamental to task completion (Jehn, 1997), actors in OCs tend to present heterogeneous knowledge backgrounds, varied goals and needs, and potentially contesting views (Barrett et al., 2013; Benkler and Nissenbaum, 2006; Faraj et al., 2011; Wasko and Faraj, 2005; von Krogh et al., 2012). Such heterogeneity reflects in diverse contributions, which is fundamental for the success of OCs as knowledge-creation spaces (e.g. Benkler, 2002; Faraj et al., 2016; Benkler and Nissenbaum, 2006). However, at the same time, diversity in contributions also leads to contestation and disagreement amongst members and inconsistencies amongst their contributions (Faraj et al., 2011; Shaikh and Vaast, 2016). Recent research shows that people that collaborate through information technology (IT) platforms tend to experience higher levels of conflicts, which deteriorates such teams performance (Hinds and Bailey, 2003). Despite the fact that conflicts and respective impact in traditional organizations have been intensively studied (e.g. Greer et al., 2008; Hinds and Bailey, 2003; Jehn, 1997; Rahim, 2017), the same is not true for open organizations with open access and flat structures, such as OCs.

OCs are a projecting example of open forms of organizing with a high potential to generate valuable contribution that might even significantly outperform traditional organizations (Baldwin and Von Hippel, 2011; Benkler, 2006; Benner and Tushman, 2015; Faraj et al., 2016; O'Mahony and Lakhani, 2011). However, mainstream managerial literature is unable to inform us about how such open organizations, without formal structure, authority, or formal pipeline, 
are able to coordinate contesting contributions from heterogeneous participants (Boudreau and Lakhani, 2015; Dahlander and Frederiksen, 2012; Franke and Shah, 2003). Existing research defend that communities' features such as reputation, status, affiliation, and stratified roles constitute informal mechanisms that facilitate task division, allocation, and coordination (Dahlander and Frederiksen, 2012; Lee and Cole, 2003), facilitating learning and knowledge creation in OCs (e.g. Dahlander and Frederiksen, 2012; Lee and Cole, 2003). However, less effort has been devoted to understanding the processes and mechanism allowing OCs to coordinate contesting contributions from multiple heterogeneous members with distinct visions and knowledge backgrounds.

Our research studies two OpenStreetMap communities in Belarus and Portugal and builds on the analysis of 100 cases of contesting member contributions discussed at the community forums, chats and mail lists (300 pages of text), 30 in-depth interviews, as well as 16 hours of ethnographic observations of offline community meetings and informal peer-to-peer discussions.

The findings reveal that contributing $\mathrm{OC}$ actors self-organize into two groups that use diverse ecosystem of tools: while content group relies mainly on existing tools embedded into the OSM platform the expert group intensively use a variety of external and self-created tools in addition to OSM tools. The above differences in the ecosystem toolkit intensify contesting contributions from different members and, particularly, between groups. At the same time, the two groups engage in different sets of contribution mechanisms. The interplay between the groups and their diverse contribution mechanisms helps the community to learn via developing and implementing new tools for editing and visualizing contesting contribution, thus improving overall quality of the map. Based on our findings, we develop a process model of transforming contesting contributions into productive outputs within OCs and discuss implications of these findings for research on open organizing and online communities.

\section{METHODOLOGY}

\section{Research Setting and sampling}

OpenStreetMap (OSM) is a community-led world-mapping project, which main goal is to create a free and editable map of the world (Wiki OSM, 2019). Launched in 2004 by Steve Coast, the project grew on an impressive scale, counting more than 1 million of contributors in 2018 (OpenStreetMap, 2019; OpenStreetMap Blog, 2019). OSM provides a particularly suitable setting for researching on how open organizations manage contesting and conflicting contributions because is open project where "anybody can enter anything they wish" (Wikipedia, 2019), and where contributions are highly diverse in terms of layers of the map and edits, what generates potential for conflicts and contestation. Additionally, OSM contributions are well documented at the OSM platform and in local community forums, chats, and email lists, contributing to data richness.

Since OSM is a worldwide community formed by smaller country based communities, we focused on Belarus and Portugal OSM communities for our research setting. OSM communities in Belarus and Portugal share all the typical features of OCs: they have an open-content license and "bring together large numbers of geographically dispersed individuals in support of an activity, interest or identity" (Faraj et al., 2016, p. 668). Additionally, they build on contributions from multiple heterogeneous actors of a diverse nature (e.g. individuals, government and nongovernmental organizations) with highly diverse motivation and participation dynamics (e.g. 
Benkler, 2002; Benkler and Nissenbaoum, 2006; Faraj et al., 2016; Faraj et al., 2011; Haefliger et al., 2011). Additionally, OSM communities in Belarus and Portugal are compatible in several important dimensions, including the number of registered members $(9,515$ in Belarus and 10,744 in Portugal), the speed of mapping ${ }^{1}$ (6,208 in Belarus and 5,711 in Portugal), and the dates of first OSM edits (2007 in Belarus and 2006 in Portugal) (OSM Country Statistics, 2019).

\section{Data collection and sources}

This paper explores several data sources, which allows for data validation and triangulation, providing a stronger basis constructs and relationships (Yin, 2006). The data sources are in-depth interviews, archival records, and ethnographic observations. For the interviews, we sent interview requests through the OSM messenger to top and bottom contributors (based on the rankings from the national statistics) of the OSM projects in Belarus and Portugal. We conducted 30 interviews either face-to-face or via Skype or Viber. We taperecorded and transcribed verbatim the interviews in the native/preferred language of the interviewee (Belarusian, Russian, Portuguese or English). To the interview data, we added archival records from public OSM forums, chats, and email lists. Such archival data allowed us to analyse cases of arguable and contested edits performed by different OSM members. This screening resulted in more than 300 pages of data with 100 cases of discussions about arguable and contested edits. The last piece of data collected comprehends ethnographic observations. The first author attended offline educational and special events (e.g. OSM anniversaries) at the community meetups and informal "beer" gatherings. The author also followed educational lectures and discussions on important arguable topics, shadowing member discussions during breaks between the planned activities to get insights about the ongoing important and contentious issues in community activities and self-organizing, the variety and scope of diverse actors involved, etc.. In total, we covered 16 hours of events.

\section{Data analysis}

The data analysis followed a three-step qualitative methodology based on a thorough analysis of the interview transcripts, archival records, and ethnographic observations. We began by creating in-vivo (Gioia, Corley, and Hamilton, 2013; Glaser and Strauss, 1967) empirical themes using a language as similar as possible to our informants. The next stage of analysis drove the identification of conceptual categories grounded on empirical evidence and theoretical literatures, which involved an increasing level of interpretation (see Miles and Huberman 1994). We regularly discussed and challenged second order concepts until reaching consolidation and stability. We then further theorized second order concetps developing aggregate categories. Simultaneously, we compared the findings with the literature ensure a coherent dialogue between theory and data. Figure 1 illustrates the interplay between conceptual and aggregate dimensions through a model.

Figure 1 about here 


\section{FINDINGS}

Our findings reveal the processes that enabled OSM community to coordinate multiple and conflicting contributions from heterogeneous members with distinct visions and knowledge levels (see Figure 1).

\section{Producing contested contributions}

Our findings identify that contested contributions emerge around two types of edits: new contributions and changes on existing contributions (see Figure 1). New contributions are often subject to conflicting visions, or because members do not comply with existing norms, or because there is no standards norms. Despite the fact that OSM Wiki provides general editing recommendations, it is indeed very difficult to predict every edition and insertion on the map, what may generate blind points in the norms. Additionally, members' opinion about the "truth of the ground" might be subjective and diverse due to the different knowledge levels, experience, data sources, and ideological positions. Since OSM presents a virtual flat structure, which means that all members, no matter their seniority or experience, can change each other edits on the map; existing edits are often subject to intended or unintended transformations/edits by other members. Unintended changes include cases when unexperienced members modify or delete existing objects on the map by mistake, or when there is incompatibility amongst different OSM IT platforms or tools. Intendent changes include various cases of vandalism as well as deletion of objects coming from private or suspicious data sources by the OSM members. Thus, vandals transform existing objects for fun and/or because of ideological motives, such as re-naming the streets into Belarusian language only (deleting the pairing titles in Russian), or renaming the House of Parliament as "Daddy's house." Therefore, contested contributions emerge through new or existing contributions that are intentionally or unintentionally edited by OSM members.

\section{Self-organizing groups with diverse ecosystem of IT tools and sets of contribution mechanisms}

Our findings illustrate that contributing members self-organize in two groups (level of engagement) who use diverse ecosystem of tools and rely on distinct sets of contribution mechanisms (see Figure 2). Content providers make edits by using existing OSM IT platforms and tools, communicating via embedded OSM messenger. In contrast, members from the expert group use a wide variety of IT tools (OSM tools but also Telegram, email lists, blogs, bots, plugins, neural networks, as well as drones and cameras); engage in offline meetups, connecting to other communities; and create new IT tools for editing and improving the map and also new communication and coordination tools.

Using diverse ecosystems of tools

OSM community relies on a variety of IT tools to create and manage members' contributions (see Figures 1). Such tools include a variety of editing tools such as ID, JOSM, OsmAnd, OSRM; communication tools such as OSM messenger blogs and user diaries; knowledge repositories; and change set tools. Many members, however, go beyond the above OSM ecosystem tools and use external and self-created tools. External tools include popular 
social media and communication tools (e.g. Facebook groups, Telegram, mail lists, etc.); various data collection tools; as well as drones and web cams. Members also create new tools as personal websites and blogs devoted to OSM; statistics and data visualization tools; and a variety of commercial and non-commercial apps (e.g. Maps.me, Karta.by). In addition, OSM members create pieces of code, scripts, neural networks, and bots, implementing those codes at the OSM platform.

Our analysis shows that content providers rely mainly on OSM tools. In contrast, members from the expert group intensively use a variety of external and self-created tools in addition to OSM tools. The above differences in the ecosystem toolkit intensify contesting contributions from different members and, particularly, between groups.

\section{Relying on diverse sets of mechanisms}

Our results show that there is a range of mechanisms that members can use to contribute to the OSM community. The mechanism of contribution by initiative involves contributing with new edits on the map and controlling quality of own data and contributions. The peer-to-peer mechanism contribution involves co-shaping norms of editing via disputes, educating other members on how to use different tools and pieces of codes, coordinating, and motivating other members. Both content providers and experts both mechanism.

In addition to these contribution mechanisms, experts use a range of other types of contributions. They use control by IT, which encompasses the verification of edits by others with bots and pieces of code (scripts), following the authorship and history of edits, and coordinating inputs via external communication tools as Telegram. Finally, experts often use the role taking mechanism, which encompass taking the lead in discussion groups that reflect on new edits meanings (e.g. road signs and toll roads for local residents and foreigners, local rules for residents of border districts, etc.), acting as brokers for the community to enable problem solving using their social ties.

\section{Interplay between groups and generation of productive outputs}

Content providers doubts, discussions, and arguments about ways of mapping stimulates experts to synthesize multiple points of view, and build on constructive criticism (Greer et al. 2008; Jehn, 1997; Pelled et al., 1999). Experts also tend to be open and eager to correct wrong edits and teach these contributors how to edit rightly, as well as to promote and increase OSM community. The interplay between groups also helps the community to learn via developing and implementing new tools for editing and visualizing contesting contribution, improving overall quality of the map. Likewise, experts, stimulated by content providers, are regularly checking the validity and correcting wrong inputs, engaging in disputes about norms and ways of mapping, educating members, and attempting to socialize content providers (see Figure 1). Socialization of content providers, in particular, helps the community to decrease contesting views and develop a common vision. In summary, interactions between content providers and experts is emergent and highly productive, as it stimulates contributions, activities, and engagement of both groups to go beyond maps edits, generating an inter-group constructive process (see Figure 1).

Contesting contributions from content providers and experts resulted in a variety of productive outputs. Such outputs include edits on the OSM map but also norms and standards of mapping, education, tools and verification of edits' quality. Tools concerns the OSM members communication tools (e.g. Telegram forum); new tools for data visualization; mapping and 
editing; and data that constitute an important toolkit for the community to identify, verify, modify and coordinate contesting member contributions. Norms and standard of mapping that result from contesting contributions, enable high quality map to exist and be regularly updated. New norms and standards emerge via monitoring communication and behavioural input online, discussing at offline meetings, and community events possible alternatives and options.

Education is another output that result from the group interplay around contesting contributions, which further extends the community ecosystem of available IT tools and diversity of mechanisms to deal with contesting contributions. Finally, verification of quality consists of checking the validity and quality of multiple and contesting contributions. Verification allows checking the validity of edits and eliminating wrong ones (e.g. created by vandalism), playing a key role in enabling the quality of the collective community-generated map. These outputs enhance mutual effectiveness and enable the generation of high quality and comprehensive maps, what is the final goal of OSM community.

\section{DISCUSSION AND CONCLUSIONS}

Our findings reveal how open forms of organizing, such as OSM community, transform and coordinate contesting contributions from multiple heterogeneous members with competing visions and knowledge levels into productive outputs. As our process model illustrates, OCs tend to self-organizes into groups that use diverse ecosystem of IT tools and contribution mechanisms, engaging into conflicting-productive interplay. Such interplay, however, generates constructive processes within and between groups and enables these to transform contesting contributions into a variety of productive outputs.

Our findings provide important contributions to the studies of open organizing and OCs, specifically, about the role of contestation in such organizations. In contrast to extant research about the destructive role of conflicts in teams, specially, those operating within IT-mediated contexts (e.g. Greer et al., 2008; Hinds et al., 2003; Rahim, 2017); our findings highlight the dual role that IT tools have in OCs. On the one hand, the use and creation of diverse IT tools with different capabilities can lead to confusion, contestation, and conflicts amongst members. On the other hand, IT tools are important to facilitate the generation of productive outcomes, such map quality and scope, within IT-mediated OCs context.

Furthermore, our findings support previous studies about organizational mechanisms that enable OCs to segregate into distinct groups to coordinate and govern heterogeneous member contributions (e.g. Dahlander and Frederiksen, 2012; Shaikh and Vaast, 2016). We extend these findings by illustrating that such self-organised groups contribute in different ways to the community, using or creating different sets of IT tools. We also add to the literature that the interplay between these groups and consequent contestation is at the heart of the development and proliferation of open forms of organizing.

\section{REFERENCES AVAILABLE FROM THE AUTHORS}

Figure 1

Processes Transforming Contesting Contributions into Productive Outputs

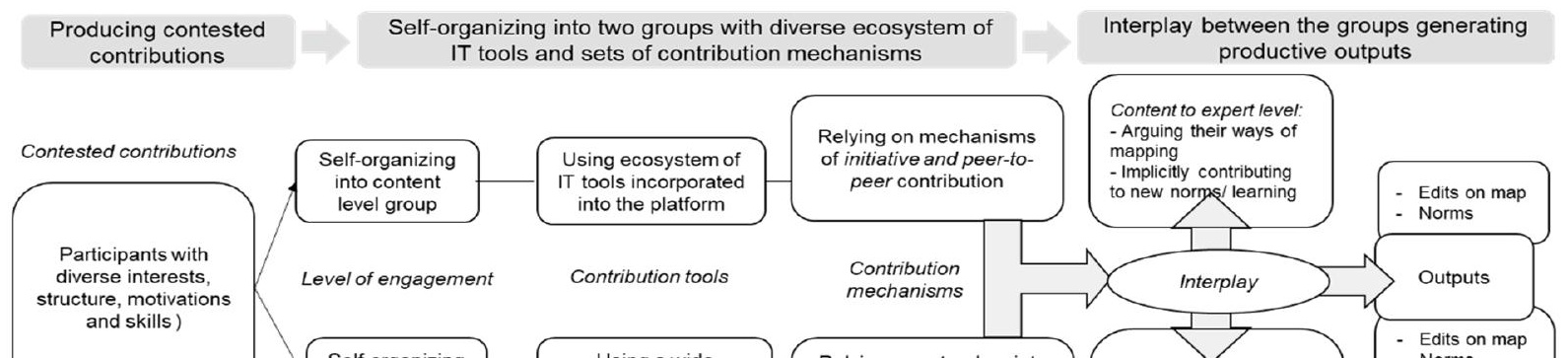


Copyright of Academy of Management Annual Meeting Proceedings is the property of Academy of Management and its content may not be copied or emailed to multiple sites or posted to a listserv without the copyright holder's express written permission. However, users may print, download, or email articles for individual use. 